\title{
Research on the Trends of Work Collaboration Industry Under Covid-19
}

\author{
Danyang Chen ${ }^{1}$
}

${ }^{1}$ New York University, New York City, NY, 10003, United States
${ }^{*}$ Corresponding author. Email: dc4594@nyu.edu

\begin{abstract}
Due to covid-19, there is a general shift in the work collaboration industry. This essay will firstly introduce the direction that the work collaboration industry is moving to under the pandemic. Then, it will provide several predictions about how the market will shift in the future. To achieve a reliable conclusion, a market landscape map of the collaborative software market will be constructed, including unified communications, file sharing and synchronization, documentation, portals and intranet as well as social collaboration. Based on that, current status can be identified from the category, functions, and technology of work collaboration platforms. Firstly, the traffic in unified communication category, video conferencing surges due to the pandemic. Secondly, there is a new need to focus on platforms with unified and simplified functions. Thirdly, to support unification and simplification, high quality and affordable technologies are required including AI, AR and VR. Finally, it leads to a reasonable prediction that the work collaboration industry is moving towards a new direction - personalization, which includes providing customized services with a more specified and professional function as well as more secured confidential protection.
\end{abstract}

Keywords: Work Collaboration, Digital Platform, Augmented Reality and Visual Reality, Work Efficiency, Covid-19.

\section{INTRODUCTION}

As Covid-19 broke out from 2020, working remotely is becoming a common trend forced by continuing lockdown. To make a quick reaction, organizations are required to set up communication and collaborations to ensure efficiency and productivity. Such a sudden rush of urgent needs brought work collaborations industry into a new direction. Under the pandemic, in order to improve the efficiency of workforce, the need towards unified communications, file sharing and synchronization, documentation, portals and intranet, as well as social collaboration boosts, which then shifts the collaboration market to a new direction. A 2020 Gartner survey reported $82 \%$ of business leaders intended to allow remote working some of the time while $47 \%$ of them intended to conduct remote working on a full-time basis [1]. However, a prediction of what changes to the market should be expected in and after the pandemic still remains to be discussed. Therefore, this essay aims to find out the new direction of work collaboration industry under the effect of Covid-19, including the reasons and methods of heading to personalization. To draw a reliable conclusion, the research will build up a market landscape map of the collaboration market, from which the scientific data and real cases can be derived. Based on such analysis, it can lead to a persuasive prediction about the collaboration trends under the effect of the pandemic as it is likely to continue, which can help corporates to improve collaboration and productivity under the uncertainty.

\section{THE STATUS OF THE WORK COLLABORATION INDUSTRY UNDER THE EPIDEMIC}

\subsection{Category}

Within the collaboration industry, one of the biggest winners is video conference platforms such as Zoom, Microsoft Teams, Hopin At the start of lockdown, corporates were dedicated to finding cloud-based unified communication tools, in which video conferencing plays an outstanding role to connect co-workers. Also, it makes centralize communication possible between in-house and remote employees via desktop and mobile devices [2]. 
Under such circumstances, the traffic of video conferencing surges as a result of the lockdown. It is reported that in 2019, $45 \%$ of employees used video conferencing tools on a daily or weekly basis [3]. However, by April 2020, the implementation of video conferencing tools increased to $58 \%$. Specifically, Cisco usage has increased by $600 \%$, Zoom by $350 \%$, Microsoft Teams by $300 \%$ [4]. To exemplify, one of software companies Hopin, has developed its function of social network and video conferencing as a live virtual event platform at the startup. To conclude, Covid-19 brought significant increasing in the need for video conferencing platforms, which is developing rapidly in the present.

\subsection{Function}

As the lockdowns continue, many businesses turn to look for a more unified platform with more advanced tools to refine their remote work strategy, establish a more seamless collaboration between vendors and customers, and thus, improve efficiency [5]. In the meantime, corporates are dedicated to replicating the traditional ways of communication across teams and departments, whereas the informal chats that can be conducted in the office is hard to be replicated online [6]. Therefore, it requires collaboration platforms to bring together functions including video conferencing, meeting scheduling, content sharing, note-taking, etc. Also, they need to make the user experience as simple as possible to allow informal communication that can bring people together and ensure spontaneous collaboration.

To exemplify, IntelePeer, founded in 2003, has moved from a single communication platform to an integrated one with multi-channel communications and automation, self-service, analytics, all delivered through a single platform. It enabled them to communicate better, drive more revenue, improve their customer experience, and make better business decisions.

\subsection{Technology}

As businesses are close with cloud collaboration, they are in urge of a high-quality video conferencing technology that enables easier information-sharing and synchronization to improve remote teamwork at scale, one of which is Artificial Intelligence. AI can be used to manage and improve the meeting room and support a more specified function such as automatic transcriptions and room scheduling. Meanwhile, it can manage video with auto zooming or improve audio with human voice recognition. According to McKinsey's State of AI report in 2020, 50\% of respondents indicate that their companies have adopted AI in at least one business function. For instance, Krisp, a platform specified in muting background voices is using $\mathrm{AI}$ as their main support. They use human detection, supported by AI, which can recognize the movement of the speaker and automatically adjusts background and camera during the video conferences.

Moreover, AR and VR have become another popular trend growing fast in 2020 due to the pandemic. Specifically, AR can be used to display a huge amount of information visually in real-time while VR can significantly enhance workers' everyday workplace for better meetings as well as collaborations. Backed by AR and VR, corporates can facilitate more immersive remote meetings and create a virtual space for teams and members to visualize the workforce and ideas [7]. To demonstrate, interior designers can create a 3D mock-up of the space of their designs and modify them simultaneously. Similarly, engineers can use 3D imaging to access a more accurate sense of robotics projects. Hence, virtual meetings supported by AR/VR may be the new direction that will gradually replace video calls and become the next frontier for work collaboration market.

\section{PREDICTIONS OF WORK COLLABORATION INDUSTRY UNDER ONGOING PANDEMIC}

Though unification is a popular trend at this time, the digital collaboration is moving to achieve a new direction-making a timely behavior change, which is fundamentally driven by personalization. According to McKinsey, personalization at scale can contribute 5\%$15 \%$ increase in companies' revenue if it's done effectively.

\subsection{Personalized Functions}

As for main targets of collaboration platforms, companies at a medium to large scale always have a more urgent need of creating a central hub where their employees can discuss ideas, establish connections, and share information besides email. It is because they have a higher demand for a flatter communication structure and a faster and authentic way of engaging to ensure efficiency and productivity. Otherwise, there might be a collaboration overload which would trap workers in excessive information, and thus, stop them from completing their work productively. Deloitte reported that $47 \%$ of respondents to their Human Capital trends survey in 2018 indicated lingering concerns about the validity of a personalized platform that enables networking across teams to avoid collaboration overload.

To incorporate personalization in those medium-tolarge-scale companies, a single intranet chat channel is not sufficient. Employees need a platform that can help them sort out information they need even when they are not checking. In this way, a platform with integrated and professional functions is required to offer highly personalized experiences that can make the workforce unique. As what has been discussed before, there are already accessible technologies that can provide 
personalized services. For instance, AI can analyze customer preference as well as support a more specified function including automatic transcriptions and room scheduling. Meanwhile, AR can create a visual insight of excessive information while VR can build up a visualized workforce in real-time. Hence, it is likely that there will be increased interest in an integrated platform with more specified and professional functions. As a result, many startups turn to specify a certain function come into being. For instance, Krisp focuses on noise cancellation. It removes unwanted background noise in calls in realtime. According to Crunchbase, despite a startup, it has successfully raised $€ 7.4 \mathrm{M}$ while their active users have grown 20-fold in 2020. Therefore, such specified tools like noise-cancellation or note-taking will be another frontier in the collaboration market.

\subsection{Security}

Furthermore, with a particularly large amount of information being processed within those medium-tolarge scale companies, cybersecurity is becoming a significant issue. Hackers have greatly expanded their attacks against corporates worldwide during the pandemic, as there is a $600 \%$ increase in attacks on cloud servers reported from January to April 2020[9]. Under such circumstances, as those organizations assign most of their workforce with a remote working option, they are in urge of a more secured plan for robust protection, better compliance, and higher efficiency [6]. Specifically, they need a guarantee for the management and control of users' access and data, a securing network against malware threats such as unauthorized entering as well as encrypting communications and corporations.

To resolve this concern, AI plays an important role in voice recognition and cancelation to ensure a private and safe environment. Moreover, in terms of communication and data privacy, confidential computing is required to construct an additional layer of security especially for sensitive information. Though this technology is still in the growth stage, it is expected to be the mainstream in the future. To demonstrate, leading digital platforms such as Google, Microsoft and IBM are using confidential computing consortium to develop new protocols and practices [8]. Therefore, security is becoming more of a priority as the remote working is expanding.

\section{CONCLUSION}

To conclude, affected by Covid-19, there is a significant shifting in work collaboration industry. Firstly, among all categories including unified communications, file sharing and synchronization, documentation, portals and intranet and social collaboration, video conferencing as a branch of unified communications share a large market percentage and reach an incredible increase at present. Secondly, overall, organizations and corporates are asking for a digital platform with unification and simplicity in order to ensure spontaneous and efficient communications and collaborations. Thirdly, to achieve unification and simplicity, Artificial Intelligence, Augmented Reality and Visual Reality are in great demand, as they can provide not only high-quality and accessible support, but more specified and professional services, which is really critical for the next stage. Based on that, this essay raises a reasonable prediction, that is digital platforms are moving to the next stage--personalization. As the pandemic is expected to continue, companies will ask for a more personalized workforce experience, which requires work collaboration platforms to be more professional and integrated with more specified functions such as automatic transcript, noise cancellation and so on. Meanwhile, they are attach greater importance to security issue as a large amount of data will be processed under the continuing pandemic.

However, one thing that needs to be noticed is that most of data and cases above are derived from previous reports and articles while the real situation including the progress of the pandemic and collaboration market is changing overtime. In other words, our analysis may contain hysteresis and the prediction made accordingly may be out of date.

\section{REFERENCES}

[1] Forbes (2021) Ready Or Not: 3 Ways The Pandemic Is Reshaping The Workforce. https://www.forbes.com/sites/tmobile/2021/07/21/r eady-or-not-3-ways-the-pandemic-is-reshapingthe-workforce/?sh=28840e0b40c2

[2] Synergy Research Group (2019) Cloud Communications Continue to Drive and Disrupt the \$45 billion Collaboration Market. https://www.srgresearch.com/articles/cloudcommunications-continue-drive-and-disrupt-45billion-collaboration-market

[3] Stone, K. (2020) The State of Video Conferencing in 2020.https://getvoip.com/blog/2020/07/07/videoconferencing-stats/ (Accessed: 22 August 2021)

[4] Constantin, L. (2020) Use of cloud collaboration tools surges and so do attacks. https://www.csoonline.com/article/3545775/use-ofcloud-collaboration-tools-surges-and-so-do-theattacks-report-

shows.html\#: :text=The\%20use $\% 20$ rate $\% 20$ of $\% 2$ 0certain,\%25\%20and\%20Slack\%20by\%20200\%25

[5] Mckinsey (2020) The Next Normal The recovery will be digital.

https://www.mckinsey.com/ /media/mckinsey/busi ness $\% 20$ functions/mckinsey $\% 20$ digital/our\%20insi ghts/how\%20six\%20companies\%20are\%20using\% 
20technology $\% 20$ and $\% 20$ data $\% 20$ to $\% 20$ transform $\% 20$ themselves/the-next-normal-the-recovery-willbe-digital.pdf

[6] Zaagman, R. (2021) Our Top 3 Collaboration Trends in 2021.

https://www.worksighted.com/blog/collaborationtrends-in-2021/

[7] Seymour, S. (2020) 8 Future Collaboration Trends \& Forecasts for 2021/2022 - A Look into What's Next. https://financesonline.com/collaboration-trends/

[8] Newman, D. (2021) Top 10 Digital Transformation Trends For 2021.

https://www.forbes.com/sites/danielnewman/2020/ 09/21/top-10-digital-transformation-trends-for2021/?sh=34255fc9c6f4 